\title{
Armut im Alter - aktuelle Daten und Entwicklungen
}

Nachdem die Altersarmut in der Bundesrepublik lange Zeit kein Thema war, hat sich nun eine heftige Debatte um eine Rückkehr der Altersarmut entwickelt. Vor diesem Hintergrund geht der Beitrag zwei Fragen nach: Wie verbreitet ist die Altersarmut heute? Und: Was ist in der Zukunft zu erwarten? Besonderes Augenmerk wird dabei auf die unterschiedliche Armutsbetroffenheit, differenziert nach Regionen, Geschlechtern und Staatsangehörigkeiten, gerichtet. ${ }^{1}$

ERIC SEILS

\section{Einleitung}

Im vergangenen Jahr gab es eine große Debatte um eine Rückkehr der Altersarmut (Butterwegge 2012, S. 75, 81). Erstaunlicherweise herrschte zwischen dem Bundesministerium für Arbeit und Soziales (BMAS) und der Opposition Einigkeit darüber, dass die Altersarmut in der Zukunft zu einem voraussehbaren, ernsten sozialpolitischen Problem wird. Diskutiert wurden daher vor allem die sozialpolitischen Reformvorschläge der Parteien, welche den Anstieg der Altersarmut in den kommenden Jahrzehnten dämpfen könnten (BMAS 2012; SPD 2012). Umstritten ist hingegen die Frage, ob Altersarmut schon heute ein Thema ist. Der Wissenschaftliche Beirat beim Bundeswirtschaftsministerium argumentiert auf der Basis eines internationalen Vergleichs, dass die Altersarmut derzeit kein Problem sei und sieht keinen rentenpolitischen Handlungsbedarf (Wissenschaftlicher Beirat beim BMWi 2012). Im Gegensatz dazu wertet die Nationale Armutskonferenz die vorhandene Altersarmut bereits als ein aktuelles Problem und sieht darin den Vorboten einer Situation, in der das Alter zum größten Armutsrisiko wird (Nationale Armutskonferenz 2012, S. 18). Angesichts solch gegensätzlicher Einschätzungen zur Dringlichkeit des Problems diskutiert dieser Beitrag aktuelle Daten zur Altersarmut im Lichte der neueren wissenschaftlichen Literatur. Dabei sollen mit Blick auf die Unterschiede zwischen Regionen, Geschlechtern, Altersgruppen und
Staatsbürgerschaft die Fragen beantwortet werden, welches Ausmaß die Altersarmut heute erreicht hat und was in $\mathrm{Zu}$ kunft zu erwarten ist. Dazu wird zunächst auf die Definition der Altersarmut und die Datenlage eingegangen (Abschnitt 2 und 3). Im zweiten Schritt (4) werden Daten zur Altersarmut diskutiert. Abschließend wird ein Fazit gezogen (5).

\section{Definition}

Der Begriff „Altersarmut“ bezeichnet die Armut im Alter. Eine Definition von Altersarmut muss jedoch zunächst klären, ab wann Menschen als alt betrachtet werden und was unter Armut zu verstehen ist. In der Literatur werden zwei Altersgrenzen verwendet, die sich beide am Rentensystem orientieren: Mit dem durchschnittlichen tatsächlichen Renteneintrittsalter lässt sich eine Altersgrenze von 60 Jahren begründen (Bönke et al. 2012, S. 177). Die Zahlen der amtlichen Sozialberichterstattung auf der Basis des Mikrozensus heben dagegen auf das traditionelle gesetzliche Rentenalter von 65 Jahren ab.

1 Ich danke Edgar Kruse und Petra Hinz von der Deutschen Rentenversicherung Bund für die Bereitstellung von Daten sowie für wichtige Hinweise. 
In den letzten Jahren hat sich die Armutsdefinition der Europäischen Union (EU) durchgesetzt. Danach gelten Menschen als armutsgefährdet, die weniger als $60 \%$ des bedarfsgewichteten Medianeinkommens zur Verfügung haben. Diese Definition ist keinesfalls selbsterklärend. Und sie hat zudem bedeutende Implikationen für die Messung der Altersarmut. Deswegen bedarf es einiger Erläuterungen:

Zunächst einmal stellt die Definition auf das verfügbare Nettoeinkommen ab. Das ist das Bruttoeinkommen nach Abzug von Steuern und Sozialversicherungsbeiträgen zuzüglich Transferzahlungen. Es geht bei der EU-Definition also um Einkommensarmut. Vermögen wird in dieser Definition nicht direkt berücksichtigt, obschon es gerade für die Wohlfahrt der Älteren von Bedeutung sein kann (Goebel/ Grabka 2011). Während im Falle von Geldvermögen die daraus resultierenden Zinseinkünfte bei der Einkommensmessung erfasst werden, ist Immobilienvermögen für die Armutsmessung weitaus problematischer. Immobilienvermögen wirft im Falle der Eigennutzung nämlich kein monetäres Einkommen ab und trägt doch zum Wohlstand des Haushaltes bei. Aus diesem Grunde wird der Mietwert selbst genutzten Wohneigentums oft als Einkommen angesehen, wenn der Datensatz dies erlaubt (Bönke et al. 2012, S. 176).

Das Medianeinkommen ist in einer nach der Höhe geordneten Verteilung das mittlere Einkommen. Es hat gegenüber dem durchschnittlichen Einkommen bei der Armutsmessung den Vorteil, dass es weniger von „Ausreißern“ beeinflusst ist. Nach den neuesten Daten des Mikrozensus für 2011 liegt das bedarfsgewichtete Medianeinkommen bei einer alleinstehenden Person bei 1.413,33 €. Die Armutsschwelle, ${ }^{2}$ unterhalb derer eine solche Person als arm gilt, liegt bei $60 \%$ dieses Betrages also bei $848 €$.

Bei der Armutsmessung wird davon ausgegangen, dass die Haushaltsmitglieder gemeinsam wirtschaften. Aus diesem Grunde werden die Einkommen aller Haushaltsmitglieder addiert. Um Haushalte unterschiedlicher Größe und Altersstruktur vergleichen zu können, müssen sie mithilfe eines Gewichtungsschemas („Äquivalenzskala“) standardisiert werden. Verschiedene Äquivalenzskalen unterscheiden sich darin, welche Einsparungen sie vom gemeinsamen Wirtschaften erwarten und welchen Bedarf sie Kindern zubilligen. Die gebräuchlichste Äquivalenzskala, auf der alle Armutsquoten dieses Beitrages basieren, ist die „modifizierte“ oder „neue“ OECD-Äquivalenzskala. Sie unterstellt größere Einspareffekte als die alte OECD-Skala. Infolgedessen ergeben sich bei Anwendung des neuen Gewichtungsschemas für Personen in größeren Haushalten höhere Wohlstandsniveaus als nach der alten OECD-Skala. Legt man die neue OECD-Skala zugrunde, fallen die Armutsrisikoquoten von Mehrpersonenhaushalten also niedriger aus als bei Verwendung der alten OECD-Skala. Daraus ergibt sich ein Effekt auf die Altersstruktur der Armut. Kinder leben normalerweise in Mehrpersonenhaushalten, ihr Armutsrisiko fällt bei Verwendung der neuen OECD-Skala rechnerisch niedriger aus als bei Nutzung der alten Skala. Alte wohnen hingegen oft in Einpersonenhaushalten. Ihr Armutsrisiko wird durch den Wechsel von der alten zur neuen Skala angehoben (MAIS-NRW 2012, S. 75).

\section{Datenquellen}

Die notwendigen Daten für die Messung der Altersarmut können im Prinzip aus jeder Haushaltsbefragung gewonnen werden, die Daten zum Einkommen erhebt. In Deutschland eignet sich eine ganze Reihe von Surveys für die Armutsmessung. Im Folgenden wird daher kurz auf die Eigenschaften der Haushaltsbefragungen eingegangen, auf die in diesem Beitrag Bezug genommen wird:

\subsection{Mikrozensus}

Der Mikrozensus (vgl. Gerhardt et al. 2009; Stauder/Hüning 2004) ist eine amtliche Haushaltsbefragung, an der jährlich etwa $1 \%$ aller Menschen in Deutschland teilnehmen muss. Dies sind etwa 380.000 Haushalte und rund 800.000 Personen. Aufgrund der enormen Größe der Befragung liefert sie auch bei tiefer regionaler Gliederung zuverlässige Ergebnisse. Die Auskunftspflicht bewirkt, dass sich Verzerrungen durch Verweigerung der Teilnahme in engen Grenzen halten. Die Befragung erfasst somit alle Einkommensgruppen und Menschen unterschiedlicher Nationalität. Diesen enormen Vorteilen steht der Nachteil gegenüber, dass der Mikrozensus das Einkommen nur global erfasst. Dadurch werden kleine und unregelmäßige Einkommen von den Befragten leicht vergessen (Stauder/Hüning 2004). Aus diesem Grunde wird das Einkommen im Mikrozensus systematisch unterschätzt. Dies hat Implikationen für die Altersstruktur der Armut. Alleinstehende und Menschen mit geringem Einkommen sind gewöhnlich besser über ihr Haushaltseinkommen informiert als Personen, die in einem Mehrpersonenhaushalt leben. Zugleich weisen diese Gruppen eine große Überlappung mit der Gruppe der Alten auf. Die Alten haben im Unterschied zur Bevölkerung im erwerbsfähigen Alter relativ viel Zeit, um über ihre Einkünfte Buch zu führen. Bei faktisch gleichem Einkommen werden die Alten daher präzise Auskünfte über ihr Einkommen geben können, während die Haushalte im erwerbsfähigen Alter ihr Einkommen vielfach unterschätzen. Dies führt dann zu einer Unterschätzung der Altersarmut (ebd. S. 27ff.). Dem steht jedoch entgegen, dass die Armutsmessung auf der Basis des Mikrozensus den Mietwert selbstgenutzten Wohneigentums nicht berücksichtigt, was tendenziell zu einer Überschätzung der Altersarmut führt. Die globale Einkommenserfassung und die Vernachlässigung des Mietwerts haben zusammen den Effekt, dass die Armutsgrenze beim Mikrozensus mit $848 € \mathrm{im}$

2 Armutsschwelle und Armutsgrenze bedeuten dasselbe. 
Jahre 2011 deutlich niedriger ausfällt als bei anderen Erhebungen. Die resultierenden Armutsgefährdungsquoten bleiben jedoch verlässlich (Stauder/Hüning 2004).

\subsection{EU-SILC-Survey}

Hier handelt es sich um eine europaweite Haushaltsbefragung, die in Deutschland unter dem Titel „Leben in Europa" läuft. Die Erhebung wird in über 30 europäischen Ländern in ähnlicher Weise durchgeführt und weist somit den Vorteil international standardisierter Daten auf. Die SILCStichprobe umfasst jährlich etwa 14.000 Haushalte. Es handelt sich um eine freiwillige Befragung, was zu der bekannten Unterrepräsentanz besonders niedriger und hoher Einkommen in der Stichprobe führt. Menschen aus diesen Einkommensgruppen neigen dazu, die Teilnahme an Haushaltsbefragungen zu verweigern („Mittelschichtbias“), was für die Armutsmessung ein offensichtliches Problem darstellt. Aus diesem Grunde erfolgt die Hochrechnung unter Berücksichtigung von Ergebnissen des Mikrozensus. Regionale Ergebnisse sind nur eingeschränkt möglich, weil die Stichprobe relativ klein und die Hochrechnung für die Bundesebene optimiert ist (Gerhardt et al. 2009, S. 9; Statistische Ämter des Bundes und der Länder o.J.). Ein weiteres Problem für die Armutsmessung mit diesem Instrument stellt die massive Untererfassung von Ausländern dar, welche bei der Hochrechnung nicht korrigiert wird. Demgegenüber ist die umfassende und detaillierte Erhebung des Haushaltseinkommens eine Stärke des Surveys. Auch der Mietwert selbstgenutzten Wohneigentums wird erfasst (Gerhardt et al. 2009, S. 9). Die Fragen, welche in der ersten Hälfte eines Jahres gestellt werden, beziehen sich dabei auf das Einkommen des vergangenen Kalenderjahres, weshalb die Kennziffern zur Armut zum Zeitpunkt der Veröffentlichung zwei Jahre alt sind.

\subsection{Sozio-oekonomisches Panel}

Das Sozio-oekonomische Panel (SOEP) ist eine Haushaltsbefragung des Deutschen Instituts für Wirtschaftsforschung (DIW) in Berlin, welche seit 1984 jährlich durchgeführt wird. Diese Datenquelle weist damit den Vorteil langer Zeitreihen auf. Die Stichprobe umfasst etwa 11.000 Haushalte mit über 20.000 Personen. ${ }^{3}$ Die Teilnahme an der Befragung ist freiwillig, was auch beim SOEP zu einem Mittelschichtbias führt, welcher durch eine Angleichung an Eckwerte aus dem Mikrozensus korrigiert werden soll. Aufgrund der vergleichsweise kleinen Stichprobe beschränken sich die publizierten Analysen der Altersarmut in regionaler Hinsicht auf Ost-West-Vergleiche. Die SOEP-Stichprobe umfasst eine überproportionale Zahl von Zugewanderten. Um Verzerrungen durch Sprachbarrieren so weit wie möglich auszuschließen, werden die Fragebögen in mehrere Sprachen übertragen und fremdsprachige Interviewer eingesetzt. Im SOEP wird detailliert nach Einkünften aus unterschiedlichen Rentenarten gefragt (Mika/Tucci 2006, S. 12f.,16f.).
Außerdem berücksichtigt die Erhebung den Mietwert selbstgenutzten Wohneigentums (Bönke et al. 2012, S. 176). Wie im Falle von EU-SILC beziehen sich die Fragen nach dem Einkommen jeweils auf das Bruttoeinkommen des Vorjahres. Dies hat wiederum zur Folge, dass die auf SOEP-Basis berechneten Armutsquoten zum Zeitpunkt der Veröffentlichung zwei Jahre alt sind (Bönke et al. 2012, S. 179).

\subsection{Bewertung}

Es zeigt sich also, dass die drei Haushaltsbefragungen unterschiedliche Stärken und Schwächen haben, die erstens bei der Interpretation der Ergebnisse berücksichtigt werden müssen und die zweitens zu widerstreitenden Ergebnissen führen können. In manchen Fällen kann die Interpretation von Armutsdaten durch Kennziffern aus der Mindestsicherungsstatistik ergänzt oder gestützt werden. Im Fall der Altersarmut kommen dafür die Prozessdaten der Anfang 2003 eingeführten Grundsicherung im Alter und bei Erwerbsminderung infrage. Hierbei ist jedoch zu bedenken, dass die Statistik der Grundsicherung im Alter nicht etwa Armut, sondern die Inanspruchnahme einer bedarfsgeprüften Leistung misst (BMAS 2013, S. 296). Setzt man den Grundsicherungsbezug hingegen mit Armut gleich, dann führt ein vereinfachter Zugang zu einer wachsenden und ein erschwerter Zugang zu einer sinkenden Altersarmut. Außerdem werden Personen als arm definiert, die ihren Anspruch geltend machen und deren Einkommen infolgedessen die Grundsicherungsschwelle zumindest erreicht. Demgegenüber wird die Armut derjenigen nicht erfasst, welche aus Scham, Furcht oder Unkenntnis keinen Antrag auf Grundsicherung im Alter stellen, obschon ihre Ressourcen das gesetzlich definierte Minimum unterschreiten.

Wenngleich die Statistik der Grundsicherung also gänzlich andere Sachverhalte erfasst als die Armutsmessung, ist es dennoch nicht unplausibel, dass der Grundsicherungsbezug und die Altersarmut über Regionen und Gruppen hinweg kovariieren. Insofern können die Prozessdaten der Grundsicherung im Alter die Analyse der Altersarmut innerhalb bestimmter Grenzen bereichern.

Erstens ist zu beachten, dass die Grundsicherungsquoten viel niedriger sind als die der Altersarmut. Dies ist zunächst einmal darauf zurückzuführen, dass die Grundsicherungsschwelle mit ca. $698 €$ deutlich unter den Armutsschwellen auf der Basis von Haushaltsbefragungen liegt (o. Verf. 2012, S. 115). Allerdings liegen die Quoten der Grundsicherung im Alter auch deutlich unter den SGB-II-Quoten der Menschen im erwerbsfähigen Alter. Als Erklärung für diesen Sachverhalt kann vermutet werden, dass man bei einer nicht unerheblichen Gruppe von

3 Die Zahlen basieren auf Angaben auf der SOEP-Homepage. 
Älteren zu Recht höhere Vermögen in Rechnung stellen kann (Noll/Weick 2011, S. 64f.); gleichwohl bleibt ein Rest, welcher vermuten lässt, dass eine erhebliche Dunkelziffer besteht (Becker 2012, S. 133). In der Tat hat Becker gezeigt, dass weitaus mehr als die Hälfte, womöglich sogar zwei Drittel der anspruchsberechtigten Älteren ihren Anspruch auf Grundsicherung nicht geltend machen (Becker 2012, S. 141). Grundsicherungsquoten können also nicht ohne Weiteres als Beleg dafür herangezogen werden, dass Altersarmut keine Rolle spielt. Außerdem ist beim Vergleich unterschiedlicher Bevölkerungsgruppen zu bedenken, dass diese in unterschiedlicher Weise geneigt sein können bzw. befähigt sind, ihre Rechtsansprüche auch durchzusetzen.

Bei der Analyse von Grundsicherungsdaten im Längsschnitt ist zweitens zu beachten, dass das Programm geschaffen wurde, um die verdeckte Armut zu verringern. Ein Anstieg des Grundsicherungsbezuges kann also auf einen Erfolg des Programms hinweisen. Außerdem könnte eine Veränderung des Leistungspaketes im Jahre 2005 zu einer Ausweitung des Kreises der Leistungsberechtigten geführt haben (Becker 2012, S. 129). Auch der geringfügige Rückgang der Empfängerzahlen im Jahre 2009 hängt mutmaßlich mit einer Leistungsausweitung beim vorrangig zu gewährenden Wohngeld zusammen (Statistische Ämter des Bundes und der Länder 2012, S. 28).

\section{Ergebnisse}

\subsection{Verbreitung der Altersarmut unter Frauen und Männern in Deutschland}

Die Verbreitung der Altersarmut kann relativ zu anderen Ländern und im Vergleich zu weiteren Altersgruppen beurteilt werden (Zaidi/Gaisor 2011).

Tabelle 1 zeigt, dass die Altersarmut der Männer in Deutschland mit $12 \%$ etwas weniger verbreitet ist als im Durchschnitt der EU-15 bzw. EU-27. Die Armutsgefährdungsquote der deutschen Frauen liegt mit 16,2 \% höher als die der Männer. Sie ist jedoch niedriger als die der Frauen in anderen europäischen Ländern. Vergleicht man nun die Armutsquoten der alten Männer und Frauen mit den jeweiligen Armutsquoten der Gesamtbevölkerung, dann zeigt sich ebenso, dass die Altersarmutsquoten bei beiden Geschlechtern unter denen der Gesamtbevölkerung liegen. Bei der Bewertung dieses Ergebnisses ist jedoch zu berücksichtigen, dass die Altersstruktur der Armut auch von der verwendeten Äquivalenzskala abhängt. Insgesamt deutet der Vergleich jedoch darauf hin, dass die Altersarmut in Deutschland gegenwärtig kein allgemeines Problem darstellt. Der folgende Abschnitt beschäftigt sich mit der Frage, inwiefern es sich hierbei um eine Momentaufnahme oder um einen andauernden Zustand handelt.
TABELLE 1

Altersarmut im europäischen Vergleich, Einkommensjahr 2010

Angaben in Prozent

\begin{tabular}{lcccccc}
\hline & \multicolumn{3}{c}{ Männer } & \multicolumn{3}{c}{ Frauen } \\
& DEU & EU-15 & EU-27 & DEU & EU-15 & EU-27 \\
\hline Gesamt & 14,9 & 15,8 & 16,1 & 16,8 & 17,6 & 17,6 \\
$65+$ & 12,0 & 13,9 & 13,2 & 16,2 & 18,4 & 18,1 \\
\hline
\end{tabular}

Quelle: Eurostat, EU-SILC (2011).

\subsection{Zur Entwicklung der Altersarmut in der Bundesrepublik}

Abbildung 1 zeigt für Deutschland die Armutsrisikoquoten nach Geschlecht und Altersgruppen für die Jahre 1996-2011. Auch diese Zahlen stützen den Befund, dass die Armut im Alter gegenwärtig weniger verbreitet ist als in anderen Altersgruppen und dass dies auch schon länger der Fall ist. Für die Bundesrepublik als Ganzes ergibt sich auf der Basis aller drei Haushaltsbefragungen (EU-SILC, SOEP, Mikrozensus) ein etwas geringeres Armutsrisiko der Alten relativ zur Bevölkerung insgesamt (Bieber/Stegmann 2011, S. 74). Allerdings fallen regional differenzierende Arbeiten auf der Basis des SOEP weniger eindeutig aus. So kamen Goebel/Grabka (2011, S. 6) zu dem Ergebnis, dass sich in Westdeutschland seit dem Jahr 2000 die Armutsquoten der Gesamtbevölkerung und die von Personen im Alter ab 65 Jahren auf demselben Niveau befänden. Lediglich die Alten in Ostdeutschland hatten danach niedrigere Armutsquoten. Berechnungen auf Basis des SOEP, welche den Mietwert selbstgenutzten Wohneigentums nicht berücksichtigen, führen gar zu dem Ergebnis, dass die Armutsgefährdungsquoten der Alten in Westdeutschland höher sind als die der Altersgruppen von 20 bis 54 und von 55 bis 64 Jahren. Zu beachten ist jedoch, dass dieses Ergebnis nur für Westdeutschland gilt und dass der Vergleich nur Personen ab 20 Jahren einbezieht (Noll/ Weick 2011). Auch eine Studie von Bönke et al. zeigt, dass die Rentner in Westdeutschland seit den frühen 1990er Jahren durchgehend eine höhere Armutsquote aufweisen als die Gesamtbevölkerung. Nur bei den Pensionären und in Ostdeutschland war die Altersarmut sehr viel weniger verbreitet als die Armut in der Bevölkerung insgesamt (Bönke et al. 2012, S. 183).

Abbildung 1 (obere Grafik) lässt erkennen, dass die soziale Lage der Alten durchaus einem raschen Wandel unterworfen ist. Sie zeigt, dass die Altersarmut zwischen 1996 und 2006 gefallen ist. Unmittelbar nach der Vereinigung war die Altersarmut in Ostdeutschland enorm hoch. In den folgenden Jahren stiegen die Renten in Ostdeutschland jedoch deutlich schneller als im Westen, was viele ostdeutsche Rentner aus der Armut hob, bis ihr Armutsrisiko unter das ihrer westdeutschen Pendants fiel (Bönke et al. 
ABB. 1

\section{Armutsquoten nach Altersgruppen und Geschlecht, Einkommensjahre 1996 - 2011}

Angaben in Prozent

Gesamtbevölkerung

über 70 Jahre

Gesamt

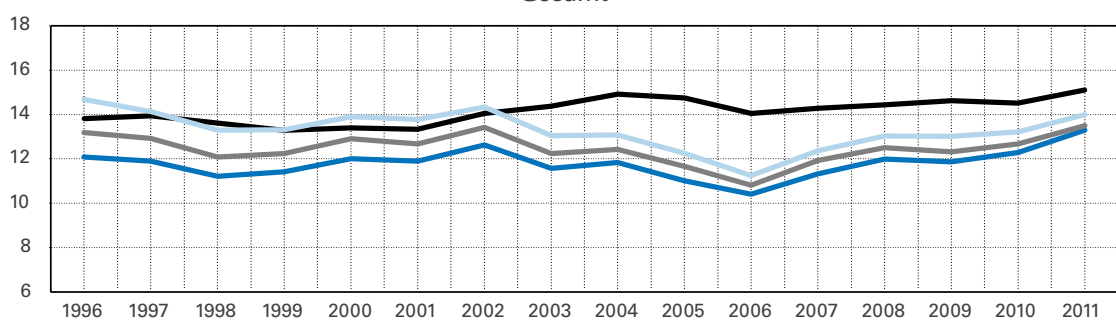

Frauen

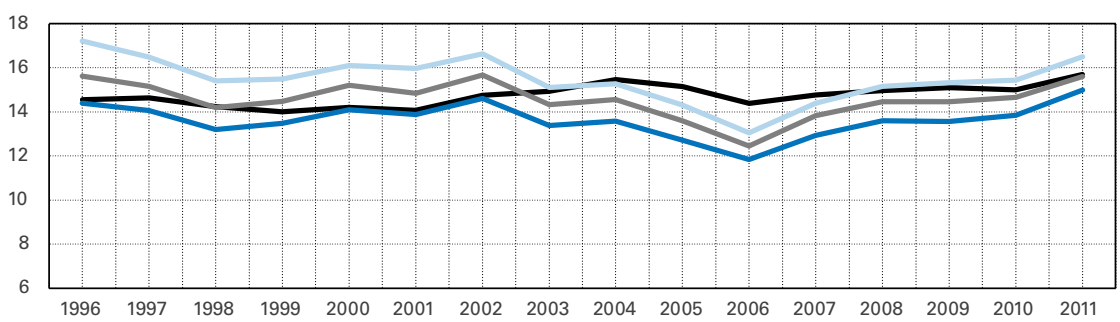

Männer

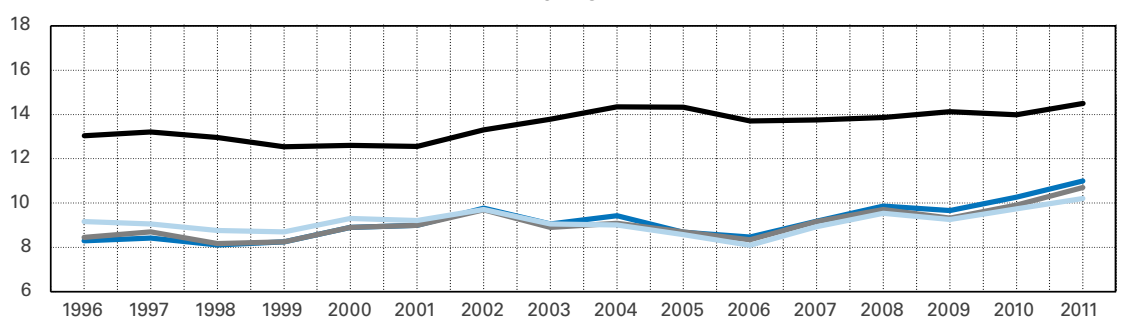

Quelle: Amt für Statistik Berlin-Brandenburg (2012), Sonderauswertung des Mikrozensus durch IT.NRW. tenanwartschaften aufbauen als Männer. Sie sind damit im Rentenalter auf die Rente ihres Mannes angewiesen, um Armut zu vermeiden. Allerdings sind Frauen bei der Eheschließung im Durchschnitt jünger als ihr Ehemann und weisen zudem eine höhere Lebenserwartung auf. Je älter die Frauen werden, desto wahrscheinlicher ist es, dass ihr Mann verstirbt und sie auf die eigene Rente bzw. auf eine Witwenrente angewiesen sind. Diese reicht oftmals nicht aus, um sie über die Armutsschwelle zu heben. Die Mikrozensus-Daten zeigen, dass der erneute Anstieg der Altersarmut dazu geführt hat, dass die Armut der Frauen über 75 Jahren schon heute den Wert für die weibliche Gesamtbevölkerung übersteigt.

Bei den Männern sind die alters- bzw. kohortenbedingten Unterschiede bei der Altersarmut hingegen deutlich geringer. Außerdem ist die Armut im Alter deutlich weniger verbreitet als in der Bevölkerung insgesamt (Abbildung 1, untere Grafik). Letzteres ist mutmaßlich ein Kohorteneffekt. Männer, die heute alt sind, haben ihre Rentenanwartschaften in Zeiten aufgebaut, in denen die Arbeitsmarktlage günstiger war als heute. Interessant ist, dass die Wahrscheinlichkeit von Armut im Jahre 1996 noch wie bei den Frauen mit dem Alter stieg. Mit dem Tod der Ehefrau verlieren nämlich auch die Männer die Einspareffekte durch das Zusammenleben. Im Laufe der Zeit hat sich die Reihenfolge jedoch umgekehrt: Heute weisen die jüngsten Rentner die höchsten Armutsquoten auf. Der Grund dürfte darin liegen, dass die jüngeren Rentner einen gröBeren Anteil ihres Arbeitslebens unter den Bedingungen einer schlechteren Arbeitsmarktlage verbracht haben. Die jüngeren Jahrgänge haben daher geringere Renten zu erwarten als die älteren Kohorten. Es zeigt sich also, dass die derzeit geringe Verbreitung der Altersarmut kein Garant für die Zukunft ist. Außerdem stellt sich die Frage, inwiefern eine allgemein niedrige Altersarmut spezifische Armutsrisiken bestimmter Regionen und Bevölkerungsgruppen verdeckt.

\subsection{Altersarmut in den Bundesländern} völkerung in der lang andauernden Rezession des letzten Jahrzehnts an, sodass die soziale Lage der Alten in diesem Zeitraum zunehmend besser ausfiel als die der übrigen Bevölkerung. Seitdem ist die Altersarmut jedoch wieder angestiegen, womit sich die Lücke wieder etwas geschlossen hat. Vergleicht man die Gesamtentwicklung mit den beiden geschlechtsspezifischen Darstellungen (Abbildung 1, mittlere und untere Grafik), ergeben sich weitere Einsichten: Erstens ähnelt die Entwicklung der Alterarmut der Gesamtbevölkerung der Entwicklung der Altersarmut der Frauen. Bei den Männern ergibt sich hingegen ein ganz anderes Bild. Der Grund dafür ist mutmaßlich, dass die Gruppe der Alten überwiegend aus Frauen besteht. Zweitens zeigt sich, dass die Wahrscheinlichkeit von Altersarmut bei den Frauen mit dem Alter deutlich ansteigt und generell höher liegt als bei den Männern. Ursächlich hierfür ist, dass Frauen aufgrund ihrer Erwerbsbiografien geringere eigene Ren-

\section{Die in Abbildung 2 wiedergegebenen Armutsquoten für die} Bundesländer zeigen, dass die Altersarmut in der Bundesrepublik sehr ungleichmäßig verteilt ist. Während in Hamburg 9,7\% der Personen ab 65 Jahren unterhalb der Armutsschwelle leben, sind es in Rheinland-Pfalz 16,8\%. Angesichts des hohen Wohlstandsniveaus von Bayern erstaunt die große Verbreitung der Altersarmut in diesem Bundesland. Möglicherweise ist dies darauf zurückzuführen, dass Bayern in der Vergangenheit zu den ärmeren Bundesländern gehörte. Die neuen Bundesländer fallen hingegen durch unterdurchschnittliche Armutsquoten der Alten auf. Nur die Altersarmut in Mecklenburg-Vorpommern erreicht das in der Bundesrepublik vorherrschende Niveau. Die gegenwärtig geringe Verbreitung der Altersarmut in den neuen Bundesländern wird auch von verschiedenen Studien auf SOEP-Basis gestützt. Der Befund gilt damit auch unab- 
hängig von der Frage, ob der Mietwert selbstgenutzten Wohneigentums in die Analyse einbezogen wird (so bei Bönke et al. 2012; Goebel/Grabka 2011; Grabka/Frick 2010) oder nicht (so bei Noll/Weick 2011).

Ursächlich für die gute soziale Lage der Alten in den neuen Ländern sind die im Vergleich zum Westen hohen Rentenzahlbeträge der Bestandsrenten wegen Alters und die insgesamt gleichere Verteilung der Rentenzahlbeträge. Gelegentlich wird vermutet, dass die im Vergleich zum Westen hohen durchschnittlichen Renten im Osten ein statistisches Artefakt darstellen (Spieler 2012, S. 134f.). Tatsächlich waren im Osten Berufsgruppen wie z.B. Ärzte in der Rentenversicherung integriert, die im Westen außerhalb derselben gestanden haben. Diese heben den Mittelwert im Vergleich zum Westen an. Allerdings zeichnet sich die Schichtung der Rentenzahlbeträge im Osten dadurch aus, dass sowohl die unteren als auch die oberen Klassen gering besetzt sind. Der Mittelwert beschreibt die Situation im Osten also trotzdem sehr gut. Im Westen sind die oberen Zahlbetragsklassen der Gesetzlichen Rentenversicherung (GRV) auch ohne Einschluss der Kammerberufe und der Beamten weitaus stärker besetzt als im Osten, weil die Löhne im Westen ungleicher sind. Zugleich gibt es im Westen viel mehr geringe Renten (Deutsche Rentenversicherung Bund 2012a, S. 38f.). Diese werden jedoch in vielen Fällen an spätere Beamte bzw. Selbstständige ausgezahlt, sodass nicht zwingend von niedrigen Renten auf Altersarmut geschlossen werden kann. Ein großer Teil sind jedoch niedrige Renten an Frauen und ehemalige „Gastarbeiter", von denen nicht alle in ihr Heimatland zurückgekehrt sind.

Trotz der gegenwärtig noch guten Lage in Ostdeutschland gibt es seit Jahren Warnungen, wonach diese Situation in Bälde in eine massive Altersarmut der Frauen und Männer in den neuen Ländern umschlagen könnte (SpiegelOnline 2008). In der Tat ist die Altersarmut den Statistiken der Sozialberichterstattung ${ }^{4}$ zufolge seit 2005 bei den ostdeutschen Männern rasch angestiegen.

Ein Blick auf Abbildung 3 zeigt überdies, dass die preisbereinigten durchschnittlichen Rentenzahlbeträge in beiden Teilen Deutschlands sinken. Gegenwärtig sind die durchschnittlichen Zugangs- und Bestandsrenten im Osten noch immer höher als im Westen. Auffällig ist jedoch, dass die ostdeutschen Zugangsrenten seit 2000 sehr schnell und kontinuierlich sinken. Die Zugangsrenten fallen dabei immer weiter unter die Bestandsrenten der Region. Dies kann als Hinweis darauf gewertet werden, dass in Ostdeutschland möglicherweise eine „Wende zur Armut“ (Spieler 2012) droht. In der Tat kommen Studien des DIW in Berlin zu dem Ergebnis, dass insbesondere die Renten ostdeutscher Männer stark sinken werden. Die Renten ostdeutscher Frauen und westdeutscher Männer bleiben danach in etwa stabil oder sinken nur wenig. Bei den westdeutschen Frauen könnte es - bei niedrigem Ausgangswert - sogar zu einem Anstieg kommen (Geyer/Steiner 2010; Simonson et al. 2012). Einer Simulationsstudie des Instituts für Wirtschaftsforschung (IW) in Halle zufolge, wird es bis zum Jahr $2023 \mathrm{zu}$
ABB. 2

\section{Altersarmut im Vergleich der deutschen Bundesländer 2011}

Angaben in Prozent

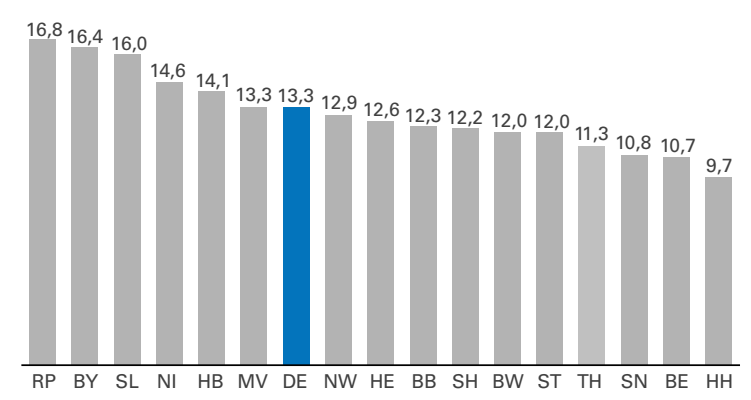

$\mathrm{BB}=$ Brandenburg, $\mathrm{BE}=$ Berlin, $\mathrm{BW}=$ Baden-Württemberg, $\mathrm{BY}=$ Bayern, $\mathrm{DE}=$ Deutschland, $\mathrm{HB}=$ Bremen, $\mathrm{HE}=$ Hessen, $\mathrm{HH}=\mathrm{Hamburg}, \mathrm{MV}=$ Mecklen burg-Vorpommern, $\mathrm{NI}=$ Niedersachsen, $\mathrm{NW}=$ Nordrhein-Westfalen, $\mathrm{RP}=$ Rheinland-Pfalz, $\mathrm{SH}=$ Schleswig-Holstein, $\mathrm{SL}=$ Saarland, $\mathrm{SN}=$ Sachsen $\mathrm{ST}=$ Sachsen-Anhalt, $\mathrm{TH}=$ Thüringen

Quelle: Statistische Ämter des Bundes und der Länder, Sozialberichterstattung (Mikrozenzsus).

ABB. 3

Rentenzahlbeträge in West- und Ostdeutschland 2000 - 2011

Durchschnittliche Zahlbeträge in Euro, Preise von 2011

Rentenzugang Rentenbestand

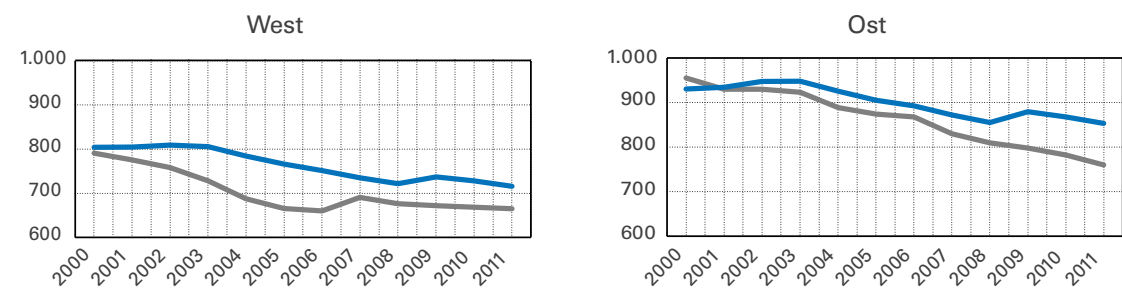

Anmerkung: Preisindex von 2010 auf 2011 umbasiert.

Quelle: Deutsche Rentenversicherung Bund (2012b, S. 125 und 203); Verbraucherpreisindex des Statistischen Bundesamtes; Berechnungen und Darstellung des Autors.

einem erheblichen Anstieg der Altersarmut kommen, welcher bei den ostdeutschen Männern drastisch ausfällt (Kumpmann et al. 2010, S. 21ff.)

\subsection{Die vergessenen „Gastarbeiter“}

In den 1960er Jahren sind von der Bundesrepublik mehrere Millionen Ausländer als sogenannte Gastarbeiter angeworben worden, um die damalige Arbeitskräfteknappheit zu mildern. Die südeuropäischen Arbeiter wurden vor

4 Die Zahlen können unter http://www.amtliche-sozialberichterstattung.de abgerufen werden. 
TABELLE 2

\section{Altersarmutsrisikoquoten und Grundsicherungsquoten nach Staatsange- hörigkeit}

Angaben in Prozent (wenn nicht anders ausgewiesen)

\begin{tabular}{l|r|r|r|r|r|r|r|}
\hline & $\mathbf{2 0 0 5}$ & $\mathbf{2 0 0 6}$ & $\mathbf{2 0 0 7}$ & $\mathbf{2 0 0 8}$ & $\mathbf{2 0 0 9}$ & $\mathbf{2 0 1 0}$ & $\mathbf{2 0 1 1}$ \\
\hline Ausländer & $\mathbf{3 9 , 7}$ & $\mathbf{3 8 , 0}$ & $\mathbf{3 8 , 5}$ & $\mathbf{3 8 , 5}$ & $\mathbf{3 8 , 6}$ & $\mathbf{3 8 , 7}$ & $\mathbf{4 1 , 5}$ \\
\hline in Tsd. & $\mathbf{1 7 1}$ & $\mathbf{1 8 0}$ & $\mathbf{1 9 5}$ & $\mathbf{2 0 7}$ & $\mathbf{2 2 0}$ & $\mathbf{2 3 3}$ & $\mathbf{2 6 8}$ \\
\hline Männer & 36,1 & 35,5 & 35,9 & 35,2 & 36,1 & 36,9 & 39,8 \\
\hline Frauen & 44,4 & 41,4 & 41,8 & 42,8 & 41,8 & 41,1 & 43,8 \\
\hline Deutsche & $\mathbf{1 0 , 2}$ & $\mathbf{9 , 5}$ & $\mathbf{1 0 , 4}$ & $\mathbf{1 1 , 1}$ & $\mathbf{1 0 , 9}$ & $\mathbf{1 1 , 3}$ & $\mathbf{1 2 , 1}$ \\
\hline in Tsd. & $\mathbf{1 5 1 6}$ & $\mathbf{1 4 5 5}$ & $\mathbf{1 6 2 6}$ & $\mathbf{1 7 4 7}$ & $\mathbf{1 7 3 3}$ & $\mathbf{1 8 0 4}$ & $\mathbf{1 9 2 7}$ \\
\hline Männer & $\mathbf{7 , 6}$ & $\mathbf{7 , 3}$ & $\mathbf{8 , 0}$ & $\mathbf{8 , 7}$ & $\mathbf{8 , 4}$ & 9,0 & 9,5 \\
\hline Frauen & 12,1 & 11,2 & 12,2 & 12,8 & 12,8 & 13,1 & 14,1 \\
\hline Grundsicherung & $\mathbf{2 , 2}$ & $\mathbf{2 , 2}$ & $\mathbf{2 , 4}$ & $\mathbf{2 , 5}$ & $\mathbf{2 , 4}$ & $\mathbf{2 , 4}$ & $\mathbf{2 , 6}$ \\
Ausländer & 13,5 & 13,1 & 13,0 & 12,9 & 2,7 & 12,8 & 12,7 \\
Deutsche & 1,8 & 1,8 & 2,0 & 2,0 & 1,9 & 2,0 & 2,1 \\
\hline
\end{tabular}

Quelle: Sonderauswertung des Mikrozensus von IT.NRW, Statistisches Bundesamt, Berechnungen des Autors.

TABELLE 3

\section{Reale durchschnittliche Zahlbeträge der Altersrenten der GRV im Inland}

Angaben in Euro

\begin{tabular}{|c|c|c|c|c|}
\hline & & 2000 & 2008 & 2011 \\
\hline \multicolumn{5}{|c|}{ Deutsche } \\
\hline \multirow[t]{2}{*}{ Männer } & Zugangsrenten & 1.196 & 1.002 & 984 \\
\hline & Bestandsrenten & 1.250 & 1.100 & 1.085 \\
\hline \multirow[t]{2}{*}{ Frauen } & Zugangsrenten & 594 & 526 & 561 \\
\hline & Bestandsrenten & 588 & 548 & 557 \\
\hline \multicolumn{5}{|c|}{ Ausländer } \\
\hline \multirow[t]{2}{*}{ Männer } & Zugangsrenten & 820 & 688 & 623 \\
\hline & Bestandsrenten & 863 & 808 & 811 \\
\hline \multirow[t]{2}{*}{ Frauen } & Zugangsrenten & 516 & 412 & 372 \\
\hline & Bestandsrenten & 545 & 483 & 478 \\
\hline
\end{tabular}

Anmerkung: Zahlbeträge in Preisen von 2011. Preisindex von 2010 auf 2011 umbasiert. schen Rentenversicherung eine unterbrochene Erwerbsbiografie auf. Derartige Wanderungen sind grundsätzlich Gegenstand zwischenstaatlicher Rentenabkommen. Allerdings war der Anteil sozialversicherungspflichtig Beschäftigter in den südeuropäischen Anwerbestaaten typischerweise niedrig (ebd.). Zweitens bedingt ihr überproportionaler Anteil an der Niedriglohnbeschäftigung und den Arbeitslosen eine im Vergleich zu deutschen Arbeitskräften niedrige Zahl an Entgeltpunkten in der deutschen Rentenversicherung. Drittens stellen die Ausländer nur einen insignifikanten Anteil an den Beamten (Statistisches Bundesamt 2012, S. 44) - also an einer Gruppe, die hinsichtlich ihrer Altersversorgung privilegiert ist (Noll/Weick 2011, S. 54ff.). 40 Jahre nach dem Anwerbestopp erreichen die einstigen "Gastarbeiter" nun das Rentenalter. Angesichts der Schwierigkeiten dieser Bevölkerungsgruppe beim Aufbau einer Alterssicherung stellt sich mit Dringlichkeit die Frage, inwiefern diese Gruppe von Altersarmut betroffen ist.

Die in Tabelle 2 abgebildeten Zahlen geben eine eindeutige Antwort: Die Quote der Altersarmut liegt unter den Ausländern derzeit bei 41,5\%. Bei den Männern sind es $39,8 \%$, bei den Frauen 43,8 \%. Die deutschen Staatsbürger ab 65 Jahren weisen hingegen eine Armutsquote von nur $12,2 \%$ auf. Die „Gastarbeiter" des Wirtschaftswunders tragen also ein enorm hohes Altersarmutsrisiko, welches zuletzt bei den Männern deutlich angestiegen ist. Dies schlägt sich auch in extrem hohen Grundsicherungsquoten der Ausländer nieder. Trotz eines geringfügigen Rückgangs in den letzten Jahren bezogen 2011 12,7 \% der Ausländer ab 65 Jahren Grundsicherung im Alter. Unter den Deutschen dieser Altersgruppe waren nur 2,1\% auf diese Leistung angewiesen. Die Altersarmutsquoten der Ausländer entwickelten sich in etwa parallel zur Gesamtbevölkerung. Allerdings ist zu beachten, dass die Zahl der Ausländer, die das Rentenalter in den letzten Jahren erreicht haben, auch deutlich gestiegen ist. In der Konsequenz hat sich die Zahl der altersarmen Ausländer von 171.000 im Jahre 2005 auf 268.000 im Jahre 2011 erhöht. Das entspricht einem Zuwachs von knapp $57 \%$. In der Gesamtbevölkerung belief sich dieser Zuwachs auf knapp $30 \%$.

Die Ursache für die massive Altersarmut unter Ausländern liegt - wie oben bereits angedeutet - in ihren geringen Rentenansprüchen begründet. Tabelle 3 zeigt, differenziert nach Staatsbürgerschaft und Geschlecht, die durchschnittlichen Zahlbeträge der Altersrenten der gesetzlichen Rentenversicherung, die ins Inland gezahlt werden. Die Beschränkung auf die ins Inland gezahlten Renten schließt kleine Renten aus, die an Personen geleistet werden, die nur einige Jahre in der Bundesrepublik gearbeitet haben und ihre Berufskarriere nach ihrer Heimkehr im Ausland fortgesetzt haben. Alle Beträge sind in Preisen von 2011 ausgedrückt, um sinnvolle Vergleiche mit früheren Zeitpunkten zu ermöglichen. Generell ist eine große Differenz der Zahlbeträge zwischen ausländischen und deutschen Männern zu beobachten. Ein alleinstehender ausländischer Altersrentner (Bestand) bezog 2011 durchschnittlich eine Rente 
von $811 €$, welche damit unter der Mikrozensus-Armutsschwelle von $848 €$ lag. Ausländischen Ehepaaren mit durchschnittlichen Bestandsrenten von Frau und Mann ist diesen Zahlen zufolge ein Renteneinkommen knapp über der Armutsgrenze beschieden. Insofern ein Blick auf die Zahlbeträge der Zugangsrenten es erlaubt, relevante Trends zu identifizieren, sieht die Zukunft düster aus: Die Zugangsrenten der ausländischen Männer sind seit dem Jahr 2000 real um fast $200 €$ gefallen. Dadurch sinken die durchschnittlichen Zugangsrenten immer weiter unter das Niveau der entsprechenden Bestandsrenten. Im Jahre 2011 lag die durchschnittliche Zugangsrente eines ausländischen Mannes mit $623 €$ deutlich unter der Grundsicherungsschwelle von $698 €$. Die Entwicklung bei den ausländischen Frauen gibt ebenfalls keinen Anlass zu vermuten, dass ihre Renteneinkommen in der Lage wären, die Verluste der Männer zu kompensieren. In den nachrückenden Jahrgängen wird die Zahl der Ausländer steigen (Fuhr 2012, S. 553). Der Ausländeranteil wird mittelfristig nur geringfügig sinken und langfristig wieder steigen. Gleichzeitig werden die älteren Kohorten mit geringem Ausländeranteil versterben. Es ist daher zu vermuten, dass Altersarmut in wachsendem Maße Ausländerarmut sein wird. Nach einem Happy End für die „Gastarbeiter“ von einst sieht es nicht aus.

\section{Fazit}

Gegenwärtig ist die Altersarmut in der Bundesrepublik sowohl im internationalen Vergleich als auch in Relation zur Verbreitung der Armut in der Gesamtbevölkerung im Allgemeinen kein verbreitetes Problem. Jedoch: Die Analyse der Entwicklung der Altersarmut in den beiden letzten Jahrzehnten hat gezeigt, dass dies keinesfalls immer so war und insofern eine Rückkehr der Altersarmut nicht ausgeschlossen werden kann. Am aktuellen Rand gibt es in der Tat Hinweise darauf, dass die Armut in der Altersgruppe ab 65 wieder ansteigt. Dies wird in Zukunft mutmaßlich vor allem die Männer im Osten treffen, die gegenwärtig sehr niedrige Altersarmutsquoten aufweisen. Und weiterhin: In all den Diskussionen um eine Rückkehr der Altersarmut ist bisher leider untergegangen, dass es mit den ehemaligen „Gastarbeitern“ schon heute eine Gruppe gibt, die in sehr hohem Maße von Altersarmut betroffen ist. Die Armutsquote der älteren Ausländer lag 2011 bei 41,5\%, die der Deutschen in derselben Altersgruppe bei $12,1 \%$. Die absolute Zahl der altersarmen Ausländer hat in den vergangenen Jahren rasant zugenommen. Auch ihr Anteil an den Altersarmen hat sich rasch ausgedehnt. Es ist damit zu rechnen, dass sich diese Trends fortsetzen.

\section{LITERATUR}

Amt für Statistik Berlin-Brandenburg (2012): Regionaler Sozialbericht Berlin und Brandenburg 2011, Berlin

Becker, I. (2012): Finanzielle Mindestsicherung und Bedürftigkeit im Alter, in: Zeitschrift für Sozialreform 58 (2), S. 123-148

Bieber, U./Stegmann, M. (2011): Aktuelle Daten zur Altersarmut in Deutschland, in: Deutsche Rentenversicherung 52 (1), S. 66-86

Bönke, T./Faik, J./Grabka, M. (2012): Tragen ältere Menschen ein erhöhtes Armutsrisiko? Eine Dekompositions- und Mobilitätsanalyse relativer Einkommensarmut für das wiedervereinigte Deutschland, in: Zeitschrift für Sozialreform 58 (2), S. $175-208$
Bundesministerium für Arbeit und Soziales (BMAS) (2012): Entwurf eines Gesetzes zur Anerkennung der Lebensleistung in der Rentenversicherung, Berlin, http:// www.sozialpolitik-aktuell.de/tl_files/sozialpolitik-aktuell/_Kontrovers/Rente67/ Referentenentwurf\%2022-3-2012 \%20RV-Lebensleistungsanerkennungsgesetz. pdf (8.5.13)

Bundesministerium für Arbeit und Soziales (BMAS) (2013): Lebenslagen in Deutschland. Der vierte Armuts- und Reichtumsbericht der Bundesregierung, Berlin: Bundesregierung, http://www.bmas.de/SharedDocs/Downloads/DE/ PDF-Publikationen-DinA4/a334-4-armuts-reichtumsbericht-2013.pdf? blob=publicationFile (8.5.13)

Butterwegge, C. (2012): Ursula von der Leyen oder: Die Wiederentdeckung der Altersarmut, in: Blätter für deutsche und internationale Politik 57 (10), S. 75-83 Deutsche Rentenversicherung Bund (2012a): Rentenversicherung in Zahlen 2012, Berlin

Deutsche Rentenversicherung Bund (2012b): Rentenversicherung in Zeitreihen, Berlin

Fuhr, G. (2012): Armutsgefährdung von Menschen mit Migrationshintergrund, in: Wirtschaft und Statistik (7), S. 549-563

Gerhardt, A./Habenicht, K./Munz, E. (2009): Analysen zur Einkommensarmut mit Datenquellen der amtlichen Statistik. Statistische Analysen und Studien, Düsseldorf, http://www.it.nrw.de/statistik/analysen/stat_studien/2009/band_58/ z089200954.pdf (8.5.13)

Geyer, J./Steiner, V. (2010): Künftige Altersrenten in Deutschland: Relative Stabilität im Westen, starker Rückgang im Osten, in: DIW Wochenbericht 77 (11), S. 2-11 Goebel, J./Grabka, M. (2011): Zur Entwicklung der Altersarmut in Deutschland, in: DIW Wochenbericht 78 (25), S. 3-16

Grabka, M./Frick, J. R. (2010): Weiterhin hohes Armutsrisiko in Deutschland: Kinder und Jugendliche sind besonders betroffen, in: DIW Wochenbericht 77 (7), S. 2-11

Kumpmann, I./Gühne, M./Buscher, H. S. (2010): Armut im Alter - Ursachenanalyse und eine Projektion für das Jahr 2023, IWH-Diskussionspapiere 2010 (8), http://www.iwh-halle.de/e/publik/disc/8-10.pdf (8.5.13)

Mika, T./Tucci, I. (2006): Alterseinkommen bei Zuwanderern, DIW Research Notes 2006 (18), http://www.diw.de/documents/publikationen/73/diw_01.c.44749. de/rn18.pdf (8.5.13)

Ministerium für Arbeit, Integration und Soziales des Landes Nordrhein-Westfalen (MAIS-NRW) (Hrsg.) (2012): Sozialbericht NRW 2012, Düsseldorf Nationale Armutskonferenz (2012): Straßenfeger. Schattenbericht der NationaIen Armutskonferenz, http://nationalearmutskonferenz.de/data/SchattenberichtSonderausgabeklein.pdf (8.5.13)

Noll, H.-H./Weick, S. (2011): Wiederkehr der Altersarmut in Deutschland? Empirische Analysen zu Einkommen und Lebensstandard im Rentenalter, in: Leisering, L. (Hrsg.): Die Alten der Welt, Frankfurt a.M., S. 45-76

o. Verf. (2012): Editorial zum Schwerpunktheft: Droht die Wiederkehr der Altersarmut in Deutschland?, in: Zeitschrift für Sozialreform 58 (2), S. 115-121

Simonson, J./Kelle, N./Romeu Gordo, L./Grabka, M./Rasner, A./Westermeier, C. (2012): Ostdeutsche Männer um 50 müssen mit geringeren Renten rechnen, in: DIW Wochenbericht 79 (23), S. 3-13

Sozialdemokratische Partei Deutschland (SPD) (2012): Altersarmut bekämpfen, Lebensleistung honorieren, flexible Übergänge in die Rente schaffen, Berlin, http://www.spd.de/linkableblob/75974/data/20120911_entwurf_rentenkonzept. pdf (8.05.13).

Spiegel-Online (2008): Vielen Ostdeutschen droht die Altersarmut, http://www. spiegel.de/politik/deutschland/niedrige-rente-vielen-ostdeutschen-droht-altersarmut-a-547118.html (14.3.13)

Spieler, A. (2012): Die Entwicklung der Alterseinkünfte in Ostdeutschland: Wende zum Besseren oder Wende zur Armut?, in: Butterwegge, C./Bosbach, G./ Birkwald, M. W. (Hrsg.): Armut im Alter, Frankfurt a.M., S. 130-143

Statistische Ämter des Bundes und der Länder (2012): Soziale Mindestsicherung in Deutschland, Wiesbaden

http://www.amtliche-sozialberichterstattung.de/pdf/Soziale_Mindestsicherung_2010.pdf (8.5.13)

Statistische Ämter des Bundes und der Länder (o.J.): EU-SILC "Leben in Europa“, http://www.amtliche-sozialberichterstattung.de/pdf/Datenquellenbeschreibung_EU-SILC_090423.pdf (8.5.13)

Statistisches Bundesamt (2012): Bevölkerung und Erwerbstätigkeit, Fachserie 1 Reihe 4.1.1, Wiesbaden, https://www.destatis.de/DE/Publikationen/Thematisch/Arbeitsmarkt/Erwerbstaetige/StandEntwicklungErwerbstaetigkeit.html (8.5.13)

Stauder, J./Hüning, W. (2004): Die Messung von Äquivalenzeinkommen und Armutsquoten auf der Basis des Mikrozensus, Düsseldorf: Statistische Analysen und Studien NRW, S. 9-31 
Wissenschaftlicher Beirat beim Bundesministerium für Wirtschaft und Technologie (BMWi) (2012): Altersarmut, http://www.bmwi.de/BMWi/Redaktion/PDF/

Publikationen/Studien/gutachttext-wissenschaftlicher-beirat-altersarmut, prop erty $=$ pdf, bereich=bmwi2012, sprache $=$ de,rwb=true.pdf (8.5.13)

Zaidi, A./Gaisor, K. (2011): Armut und Deprivation älterer Menschen in Europa.

Muster und Entwicklungstendenzen, in: Leisering, L. (Hrsg.): Die Alten der

Welt, Frankfurt a. M., S. 77-112
AUTOR

ERIC SEILS, Dr., ist Wissenschaftler im Wirtschafts- und Sozialwissenschaftlichen Institut (WSI) in der Hans-Böckler-Stiftung. Arbeitsschwerpunkte: Vergleichende Wohlfahrtsstaatsforschung, Armut und Soziales.

@eric-seils@boeckler.de 